\title{
EVALUATION OF INDICES OF GLUCOSE AND INSULIN METABOLISM IN A COORT PATIENTS WITH ANGPTL3 DEFICIENCY
}

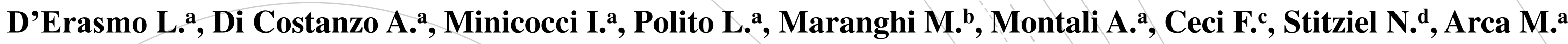

${ }^{\mathrm{a} D e p a r t m e n t ~ o f ~ I n t e r n a l ~ M e d i c i n e ~ a n d ~ C l i n i c a l ~ S p e c i a l t i e s, ~ S a p i e n z a ~ U n i v e r s i t y ~ o f ~ R o m e, ~}{ }^{\mathrm{b} D e p a r t m e n t}$ of Translational and Precision Medicine, Sapienza University of Rome, ${ }^{\mathrm{c}} \mathrm{Cellular}$ Biotechnologies and Hematology, Sapienza University of Rome, ${ }^{d}$ Department of Genetics, Washington University School of Medicine, Saint Louis, Missouri

INTRODUCTION
Angiopoietin-like 3 (ANGPTL3) is a regulator of lipoprotein metabolism by inhibiting lipoprotein lipase (LPL)
activity. In addition, it has been proposed to be able to influence insulin sensitivity. We have tested this hypothesis in
subjects carrying loss-of-function (LOF) mutations in ANGPTL3, thus presenting the familial combined hypolipidemia
phenotype.
METHODS
72 wild-type and 40 p.S17X LOF carrier subjects ( 36 heterozygotes and 4 homozygotes) have been studied for insulin
sensitivity by glucose tolerance tests (OGTT). Results in carriers and non-carrier controls have been compared.

\section{RESULTS}

Table 1 shows baseline clinical features in the whole cohort according with genotypes. Moreover, homozygotes patients were older as compared with other genotypes ( $P$ for trend $=0,045 ; P$ among genotypes=NS). Table 1

\begin{tabular}{|c|c|c|c|c|c|c|c|}
\hline & & $\%$ & Mean & SD & $\begin{array}{r}95 \% \text { Confi } \\
\text { Inferior Limit }\end{array}$ & $\begin{array}{l}\text { ce Interval } \\
\text { Upper Limit }\end{array}$ & $P$ for trend \\
\hline Age (yrs) & $\begin{array}{l}\text { Wild-type } \\
\text { Heterozygotes } \\
\text { Homozygotes }\end{array}$ & $\begin{array}{l}- \\
- \\
-\end{array}$ & $\begin{array}{l}56,4 \\
51,1 \\
66,5\end{array}$ & $\begin{array}{c}12,4 \\
16,7 \\
6,0\end{array}$ & $\begin{array}{l}\mathbf{5 3 , 5} \\
\mathbf{4 5 , 5} \\
\mathbf{5 7 , 0}\end{array}$ & $\begin{array}{l}\mathbf{5 9 , 3} \\
\mathbf{5 6 , 8} \\
\mathbf{7 6 , 0}\end{array}$ & 0,045 \\
\hline Female (\%) & $\begin{array}{l}\text { Wild-type } \\
\text { Heterozygotes } \\
\text { Homozygotes }\end{array}$ & $\begin{array}{l}50,0 \\
52,8 \\
25,0\end{array}$ & - & $\begin{array}{l}- \\
-\end{array}$ & - & - & NS \\
\hline BMI $(\mathbf{k g} / \mathbf{m})$ & $\begin{array}{l}\text { Wild-type } \\
\text { Heterozygotes } \\
\text { Homozygotes }\end{array}$ & $\begin{array}{l}- \\
- \\
-\end{array}$ & $\begin{array}{l}28,7 \\
29,4 \\
29,8\end{array}$ & $\begin{array}{l}5,4 \\
5,7 \\
2,2\end{array}$ & $\begin{array}{l}27,5 \\
27,4 \\
26,2\end{array}$ & $\begin{array}{l}30,0 \\
31,3 \\
33,4\end{array}$ & NS \\
\hline WC (cm) & $\begin{array}{l}\text { Wild-type } \\
\text { Heterozygotes } \\
\text { Homozygotes }\end{array}$ & $\begin{array}{l}- \\
- \\
-\end{array}$ & $\begin{array}{c}97,9 \\
98,0 \\
104,3\end{array}$ & $\begin{array}{c}13,5 \\
16,1 \\
5,1\end{array}$ & $\begin{array}{l}94,6 \\
92,6 \\
96,2\end{array}$ & $\begin{array}{l}101,1 \\
103,4 \\
112,3\end{array}$ & NS \\
\hline PAS (mmHg) & $\begin{array}{l}\text { Wild-type } \\
\text { Heterozygotes } \\
\text { Homozygotes }\end{array}$ & $\begin{array}{l}- \\
- \\
-\end{array}$ & $\begin{array}{l}136,9 \\
134,5 \\
130,3\end{array}$ & $\begin{array}{c}24,2 \\
16,9 \\
8,8\end{array}$ & $\begin{array}{l}131,2 \\
128,7 \\
116,3\end{array}$ & $\begin{array}{l}142,6 \\
140,2 \\
144,2\end{array}$ & NS \\
\hline PAD (mmHg) & $\begin{array}{l}\text { Wild-type } \\
\text { Heterozygotes } \\
\text { Homozygotes }\end{array}$ & $\begin{array}{l}- \\
- \\
-\end{array}$ & $\begin{array}{l}79,5 \\
79,3 \\
76,3\end{array}$ & $\begin{array}{c}10,1 \\
7,7 \\
7,5\end{array}$ & $\begin{array}{l}77,1 \\
76,7 \\
64,3\end{array}$ & $\begin{array}{l}81,8 \\
81,9 \\
88,2\end{array}$ & NS \\
\hline TC (mg/dl) & $\begin{array}{l}\text { Wild-type } \\
\text { Heterozygotes } \\
\text { Homozygotes }\end{array}$ & - & $\begin{array}{c}187,4 \\
165,8 \\
84,5 \\
\end{array}$ & $\begin{array}{c}35,1 \\
33,3 \\
9,3\end{array}$ & $\begin{array}{c}179,1 \\
154,4 \\
69,7\end{array}$ & $\begin{array}{c}195,6 \\
177,3 \\
99,3\end{array}$ & $<0,001$ \\
\hline HDL (mg/dl) & $\begin{array}{l}\text { Wild-type } \\
\text { Heterozygotes } \\
\text { Homozygotes }\end{array}$ & - & $\begin{array}{l}61,0 \\
54,5 \\
30,0\end{array}$ & $\begin{array}{c}15,4 \\
11,5 \\
6,2\end{array}$ & $\begin{array}{l}57,4 \\
50,6 \\
20,2\end{array}$ & $\begin{array}{l}\mathbf{6 4 , 7} \\
\mathbf{5 8 , 5} \\
39,8\end{array}$ & $<0,001$ \\
\hline Triglycerides (mg/dl) & $\begin{array}{l}\text { Wild-type } \\
\text { Heterozygotes } \\
\text { Homozygotes }\end{array}$ & - & $\begin{array}{c}101,6 \\
80,8 \\
33,3\end{array}$ & $\begin{array}{l}46,5 \\
31,8 \\
14,0\end{array}$ & $\begin{array}{l}90,7 \\
69,9 \\
10,9\end{array}$ & $\begin{array}{c}112,5 \\
91,7 \\
55,6\end{array}$ & $<0,001$ \\
\hline LDL (mg/dl) & $\begin{array}{l}\text { Wild-type } \\
\text { Heterozygotes } \\
\text { Homozygotes }\end{array}$ & - & $\begin{array}{c}105,6 \\
95,0 \\
42,5\end{array}$ & $\begin{array}{l}29,9 \\
28,8 \\
17,8\end{array}$ & $\begin{array}{l}98,5 \\
85,1 \\
14,2\end{array}$ & $\begin{array}{c}112,6 \\
104,8 \\
70,8\end{array}$ & $<0,001$ \\
\hline APO B (mg/dl) & $\begin{array}{c}\text { Wild-type } \\
\text { Heterozygotes } \\
\text { Homozygotes }\end{array}$ & - & $\begin{array}{l}94,4 \\
87,5 \\
53,8\end{array}$ & $\begin{array}{l}26,2 \\
28,5 \\
34,3\end{array}$ & $\begin{array}{l}88,3 \\
77,7 \\
-0,8\end{array}$ & $\begin{array}{c}100,6 \\
97,3 \\
108,3\end{array}$ & 0,012 \\
\hline Creatinine mg/dl) & $\begin{array}{l}\text { Wild-type } \\
\text { Heterozygotes } \\
\text { Homozygotes }\end{array}$ & - & $\begin{array}{l}0,9 \\
0,8 \\
0,8\end{array}$ & $\begin{array}{l}0,2 \\
0,2 \\
0,3\end{array}$ & $\begin{array}{l}0,8 \\
0,8 \\
0,4\end{array}$ & $\begin{array}{l}0,9 \\
0,9 \\
1,3\end{array}$ & NS \\
\hline Fasting glucose (mg/dl) & $\begin{array}{l}\text { Wild-type } \\
\text { Heterozygotes } \\
\text { Homozygotes }\end{array}$ & - & $\begin{array}{l}98,6 \\
95,4 \\
96,3\end{array}$ & $\begin{array}{l}17,4 \\
14,0 \\
12,6\end{array}$ & $\begin{array}{l}94,0 \\
90,3 \\
76,2\end{array}$ & $\begin{array}{l}103,3 \\
100,5 \\
116,3\end{array}$ & NS \\
\hline Fasting insulin (mg/dl) & $\begin{array}{c}\text { Wild-type } \\
\text { Heterozygotes } \\
\text { Homozygotes }\end{array}$ & - & $\begin{array}{c}10,1 \\
8,4 \\
6,9\end{array}$ & $\begin{array}{l}5,8 \\
3,2 \\
3,1\end{array}$ & $\begin{array}{l}8,6 \\
7,2 \\
2,0\end{array}$ & $\begin{array}{c}11,7 \\
9,6 \\
11,8\end{array}$ & NS \\
\hline
\end{tabular}

As expected, plasma lipids were different between genotypes while no differences were found in fasting glucose and insulin. Despite this, in a multivariate model including BMI, gender and age, we have shown significantly lower homeostatic model assessment of insulin resistance (HOMA-IR) and higher insulin sensitivity index (ISI) in S17X homozygotes as compared with the other genotypes (respectively $\mathrm{P}=0.011$ and $\mathrm{P}=0.05$ ) (Figure 2 and Table 2).

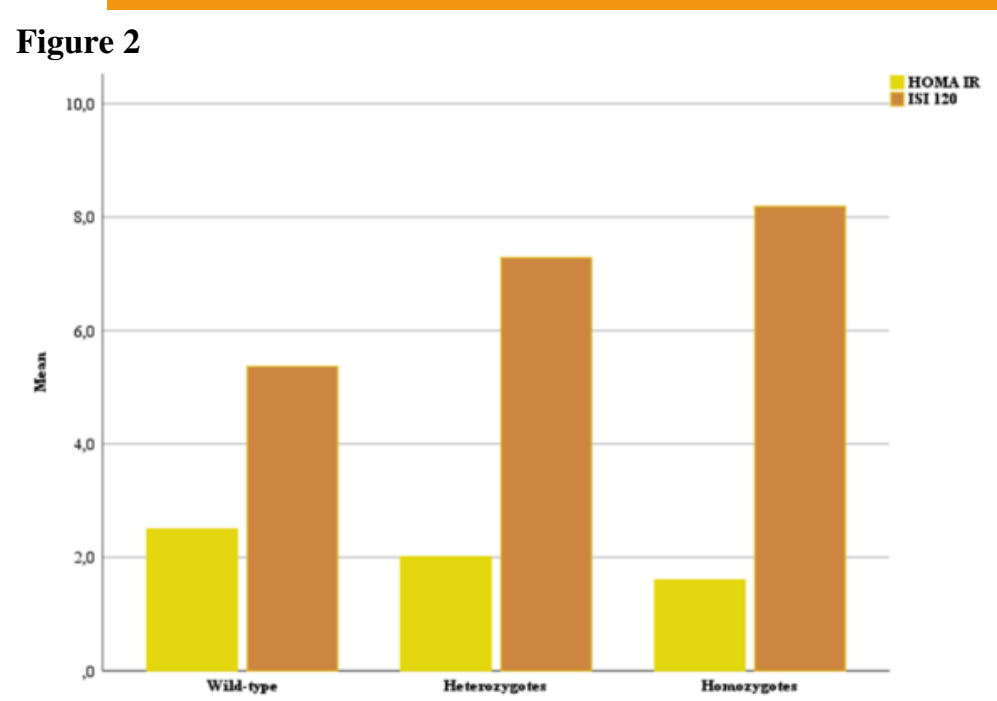

\begin{tabular}{|c|c|c|c|c|c|}
\hline & Wild-Type & Heterozygotes & Homozygotes & $\mathbf{P}$ & $\mathbf{P}_{\text {adj }}$ \\
\hline $\mathbf{N}$. & 57 & 31 & 4 & - & \\
\hline $\begin{array}{l}\text { HOMMA } \\
\text { IR }\end{array}$ & $2,5 \pm 1,6$ & $2,0 \pm 0,8$ & $1,6 \pm 0,6$ & NS & $0,011 *$ \\
\hline $\begin{array}{l}\text { ISI-120 } \\
\text { INDEXX }\end{array}$ & $5,3 \pm 3,5$ & $7,2 \pm 8,3$ & $8,2 \pm 5,2$ & NS & $0,05 * *$ \\
\hline
\end{tabular}

\section{CONCLUSIONS}

Our results indicate that the lack of ANGPTL3 is associated with improved measures of insulin sensitivity as demonstrated by lower HOMA-IR and higher ISI values in p.S17X homozygous as compared to heterozygous and wild-type carriers. The mechanism by which reduced ANGPTL3 levels lead to improved insulin sensitivity is not known so that further studies are needed to clarify this point. The beneficial effects of ANGPTL3 deficiency on both glucose and lipoprotein metabolism support the role of AGPTL3 as a potential therapeutic target. 